\title{
OPTIMAL DYNAMIC RISK CONTROL FOR INSURERS WITH STATE-DEPENDENT INCOME
}

\author{
MING ZHOU, ${ }^{*}$ Central University of Finance and Economics \\ JUN CAI, ${ }^{* *}$ University of Waterloo
}

\begin{abstract}
In this paper we investigate optimal forms of dynamic reinsurance polices among a class of general reinsurance strategies. The original surplus process of an insurance portfolio is assumed to follow a Markov jump process with state-dependent income. We assume that the insurer uses a dynamic reinsurance policy to minimize the probability of absolute ruin, where the traditional ruin can be viewed as a special case of absolute ruin. In terms of approximation theory of stochastic process, the controlled diffusion model with a general reinsurance policy is established strictly. In such a risk model, absolute ruin is said to occur when the drift coefficient of the surplus process turns negative, when the insurer has no profitability any more. Under the expected value premium principle, we rigorously prove that a dynamic excess-of-loss reinsurance is the optimal form of reinsurance among a class of general reinsurance strategies in a dynamic control framework. Moreover, by solving the Hamilton-Jacobi-Bellman equation, we derive both the explicit expression of the optimal dynamic excess-of-loss reinsurance strategy and the closed-form solution to the absolute ruin probability under the optimal reinsurance strategy. We also illustrate these explicit solutions using numerical examples.
\end{abstract}

Keywords: Absolute ruin; excess-of-loss reinsurance; HJB equation; liquid reserve; statedependent income

2010 Mathematics Subject Classification: Primary 93E20

Secondary 91B30; 91B70; 60H30

\section{Introduction}

Optimal dynamic reinsurance control problems in continuous-time risk models have attracted a lot of interest in the recent literature. However, most of the results so far, on optimal dynamic reinsurance control problems, determine optimal retention levels in a given reinsurance form such as the proportional reinsurance, the excess-of-loss reinsurance, and the combination of the proportional reinsurance and the excess-of-loss reinsurance. For instance, the optimal retention levels in a dynamic proportional reinsurance were studied by Højgaard and Taksar (1998), Taksar (2000), Schmidli (2001), and Taksar and Markussen (2003) under the optimization criterion of maximizing the expectation of the total discounted reserves until ruin or minimizing the ruin probability. In the other direction, the optimal retention levels in a dynamic excess-ofloss reinsurance were considered in Asmussen et al. (2000) under the optimization criterion of maximizing the expected discounted value of the total dividends paid until ruin in a controlled diffusion risk model. Subsequently, Zhang et al. (2007) considered the optimal combination of

Received 23 January 2013; revision received 17 July 2013.

* Postal address: China Institute for Actuarial Science, Central University of Finance and Economics, 39 South College Road, Haidian, Beijing 100081, China. Email address: mzhou.act@gmail.com

** Postal address: Department of Statistics and Actuarial Science, University of Waterloo, Waterloo, Ontario N2L 3G1, Canada. Email address: jcai@math.uwaterloo.ca 
a dynamic proportional reinsurance and a dynamic excess-of-loss reinsurance, which minimizes the ruin probability of an insurer. Luo and Taksar (2011) studied the absolute ruin minimization with optimal investment and proportional reinsurance policies. Meng and Zhang (2010) showed that a dynamic excess-of-loss reinsurance is the optimal form of reinsurance among a class of plausible reinsurance treaties under the Black-Scholes type framework of the surplus process and the optimization criterion of minimizing the ruin probability.

Most of the references mentioned above either consider a specified type of reinsurance or they model the underlying surplus risk process for an insurer by using a state-independent income process. However, in practice, most insurers have state-dependent incomes. For example, state-dependent incomes occur when an insurer invests its surplus in excess of a liquid reserve level or when an insurer's finances are such that its surplus is running short. An underlying surplus risk process with state-dependent incomes is a flexible model that is able to incorporate the different investment activities of an insurer. Therefore, to better describe the behaviors of an insurer, it is interesting to consider a model with state-dependent incomes. In addition, statedependent incomes can affect an insurer's optimal risk control policy, as illustrated in Section 5 of this paper. They also present a challenge when finding the explicit optimal risk control policy and when solving the value function in closed-form since the Hamilton-Jacobi-Bellman (HJB) equations, in this case, are usually complicated.

In this paper we want to model the underlying surplus risk process for an insurer with state-dependent incomes and obtain the explicit optimal form of dynamic reinsurance policies among a class of general reinsurance strategies. The contribution of our paper includes the following aspects. Firstly we establish a controlled diffusion model with state-dependent income for predictable càglàd reinsurance policies. Secondly we propose the optimization criterion of minimizing the probability of absolute ruin, which is a generalization of the traditional ruin probability. Within a dynamic framework we prove the optimality of the excessof-loss reinsurance for the diffusion model with state-dependent income. In addition we obtain both the explicit expression of the optimal reinsurance policy and the closed-form solution to the absolute ruin probability under the optimal reinsurance policy.

The paper is organized as follows. In Section 2, by using the diffusion approximation to the Markov jump process with reinsurance, we formulate the optimal reinsurance control problem in a controlled diffusion risk process with reinsurance. In Section 3 we prove that a dynamic excess-of-loss reinsurance is an optimal form of reinsurance among a class of general reinsurance strategies, which minimizes the absolute ruin probability. Furthermore, in Section 4 we derive the explicit expression for the optimal dynamic excess-of-loss reinsurance policy for an insurer with state-dependent income. We also give the explicit solution for the absolute ruin probability under the optimal dynamic excess-of-loss reinsurance policy. In Section 5, these explicit results are illustrated by numerical examples and some concluding remarks are given at the end of the section.

\section{A controlled diffusion risk process with a dynamic reinsurance policy}

Let $\left(\Omega, \mathcal{F},\left\{\mathcal{F}_{t}, t \geq 0\right\}, \mathbb{P}\right)$ be a complete filtered probability space on which all stochastic quantities in this paper are well defined. Here $\left\{\mathscr{F}_{t}, t \geq 0\right\}$ is a filtration that satisfies the usual conditions.

We first assume that, in the absence of reinsurance, the surplus of an insurance company follows a real-valued Morkov jump process $\left\{X_{t}\right\}$ satisfying

$$
\mathrm{d} X_{t}=\left[\delta\left(X_{t-}\right)+c\right] \mathrm{d} t-\mathrm{d} S_{t}, \quad X_{0-}=x,
$$


where $\delta(x)$, for $x \in(-\infty, \infty)$, is a nondecreasing function representing the income state of the insurer and satisfying

$$
\left|\delta\left(x_{1}\right)-\delta\left(x_{2}\right)\right| \leq L\left|x_{1}-x_{2}\right|
$$

and

$$
\delta^{2}\left(x_{1}\right) \leq L^{2}\left(1+x_{1}^{2}\right)
$$

for all $x_{1}, x_{2} \in(-\infty, \infty)$ and some $L>0$. Moreover, $S_{t}=\sum_{k=1}^{N_{t}} Y_{k}$ denotes the aggregate claims up to time $t$; here $\left\{N_{t}\right\}$ is a time-homogenous Poisson process with Poisson rate $\lambda>0$. Independent of $\left\{N_{t}\right\},\left\{Y_{k}, k=1,2, \ldots\right\}$ denote the claim sizes and are independent and identically distributed positive random variables with common distribution function $F(y)=1-$ $\bar{F}(y)=\mathbb{P}\left\{Y_{k} \leq y\right\}$, finite mean $\mathbb{E}\left(Y_{k}\right)=\mu>0$, and finite second moment $\mathbb{E}\left(Y_{k}^{2}\right)=\sigma^{2}>0$. In (2.1), $c>0$ is the premium rate per unit time collected by the insurer and satisfies a positive security loading condition $c>\lambda \mu$.

Define $M$ by

$$
M=\sup \{y: F(y)<1\} .
$$

Note that $M$ can be either finite or infinite. In the risk model (2.1), at time $t$, the income rate $\delta\left(X_{t-}\right)$ can be positive, negative, or zero, depending on the insurer's current surplus. When the surplus is negative, the insurer is allowed to borrow money to cover the deficit. Furthermore, the insurer can invest its positive surplus. With the possibility of borrowing, the insurer can continue its business when the surplus turns negative. The absolute ruin is said to occur when the net income rate is nonpositive, namely the premiums received by the insurer are not sufficient to cover its debt. Let $d$ be the largest root of the equation $\delta(x)+c=0$ for $x<0$. Because $\delta(x)$ is nondecreasing, the drift coefficient of (2.1) will always be nonpositive if the surplus drops below the critic level $d$. When this situation happens, the surplus can never return to a positive level. Mathematically, the absolute ruin is said to occur when the surplus drops below the critic level $d$. In particular, if $d=0$ then the absolute ruin is reduced to the traditional ruin. See Section 7 of Cai et al. (2009) for more details for absolute ruin.

A special case of the risk model (2.1) has been studied by Embrechts and Schmidli (1994), in which

$$
\delta(x)= \begin{cases}\alpha(x-\Delta), & x>\Delta, \\ 0, & 0 \leq x \leq \Delta, \\ \beta x, & x<0 .\end{cases}
$$

In the risk model (2.4), the constant $\Delta \geq 0$ is the required liquid reserve level, the constant $\alpha>0$ is the risk-free interest force for investment, and the constant $\beta>0$ is the interest force for borrowing. In this model, the critic level is $d=-c / \beta$. Since $-c / \beta \rightarrow 0$ as $\beta \rightarrow+\infty$, absolute ruin will reduce to the classical ruin when the interest force $\beta \rightarrow+\infty$. Therefore, absolute ruin is the generalization of the classical ruin and it is useful when evaluating the risk of an insurance portfolio with borrowing. Absolute ruin has attracted more interest in recent studies; see, e.g. Cai (2007), Gerber and Yang (2007), Borovkov (2009), Cai et al. (2009), Zhu and Yang (2008), Asmussen and Albrecher (2010), and the references therein.

In what follows we introduce the reinsurance control into the model (2.1). Assume that the insurer uses a reinsurance strategy $R$ to control its risk. Under the reinsurance strategy $R$, when the amount of a claim is $y$, the insurer will cover the amount $R(y)$ and the reinsurer will cover the amount $y-R(y)$. Here $R(\cdot):[0, \infty) \rightarrow[0, \infty)$ is a $\mathfrak{B}$-measurable function with $0 \leq R(y) \leq y$. Two important reinsurance policies are the proportional reinsurance and the excess-of-loss reinsurance. In the first case, $R(y)=a y$, where $a \in[0,1]$ is called a 
proportional retention level. In the second case, $R(y)=\min \{y, m\}=y \wedge m$, where $m \in[0, M]$ is called an excess-of-loss retention level. Here both $a$ and $m$ are constant and are independent of the loss $y$. Under a reinsurance policy $R$, we define

$$
\mu_{R}=\mathbb{E}\left[R\left(Y_{k}\right)\right] \quad \text { and } \quad \sigma_{R}^{2}=\mathbb{E}\left[R\left(Y_{k}\right)\right]^{2},
$$

where $0 \leq \mu_{R} \leq \mu$ and $0 \leq \sigma_{R}^{2} \leq \sigma^{2}$ because of $0 \leq R(y) \leq y$.

Denote by $\left\{X_{t}^{R}, t \geq 0\right\}$ the surplus process of the insurance portfolio under the reinsurance policy $R$. Then the surplus process $X_{t}^{R}$ satisfies

$$
\mathrm{d} X_{t}^{R}=\left[\delta\left(X_{t-}^{R}\right)+c-c_{R}\right] \mathrm{d} t-\mathrm{d} \sum_{k=1}^{N_{t}} R\left(Y_{k}\right), \quad X_{0-}^{R}=x,
$$

where $c_{R}$ is the reinsurance premium rate, paid continuously to a reinsurer under the reinsurance policy $R$, and $c-c_{R}$ is the net premium rate for the insurer. Here we assume that the reinsurance premium is calculated by the expected value principle with positive loading $\xi$ :

$$
c_{R}=(1+\xi) \lambda\left(\mu-\mu_{R}\right) .
$$

Throughout this paper, we consider the noncheap reinsurance, where $c<(1+\xi) \mu$, which avoids the possibility that the insurer makes risk-free money by ceding all its risks to the reinsurer. Schmidli (1994) showed that there exists a diffusion process $\left\{Z_{t}^{R}, t \geq 0\right\}$, which is a diffusion approximation to $\left\{X_{t}^{R}, t \geq 0\right\}$, satisfying

$$
\mathrm{d} Z_{t}^{R}=\left[\delta\left(Z_{t}^{R}\right)+c+\xi \lambda \mu_{R}-(1+\xi) \lambda \mu\right] \mathrm{d} t+\sqrt{\lambda} \sigma_{R} \mathrm{~d} B_{t}, \quad Z_{0}^{R}=x,
$$

where $\left\{B_{t}, t \geq 0\right\}$ is a standard Brownian motion with respect to $\left\{\mathcal{F}_{t}, t \geq 0\right\}$.

In what follows we consider the optimal dynamic control problem based on the diffusion risk model (2.5). Assume that the surplus of an insurance portfolio with reinsurance is described by the diffusion process $\left\{Z_{t}^{R}, t \geq 0\right\}$ in (2.5) and the insurer can dynamically modify its reinsurance position. For this stochastic control problem we define the reinsurance control space by

$$
U=\{g(\cdot): g \text { is } \mathfrak{B}([0, \infty)) \text { measurable with } 0 \leq g(y) \leq y \text { for each } y \in[0, \infty)\} .
$$

A reinsurance control strategy $\pi=\left\{R_{t}, t \geq 0\right\}$ is admissible if $\left\{R_{t}:=r(t, \omega, y), t \geq 0\right\}$ is a $U$-valued predictable càglàd (left continuous with right limits) process with respect to $\left\{\mathcal{F}_{t}\right\}$ and satisfies $0 \leq r(t, \omega, y) \leq y$. For a fixed simple path $\omega \in \mathcal{F}_{t}, R_{t}$ denotes the reinsurance policy applied by the insurer at time $t$, and $r(t, \omega, y)$ is the retained loss if there is a loss $y$ at time $t$. Furthermore, we define two predictable càglàd stochastic processes $\left\{\mu_{R}(t, \omega), t \geq 0\right\}$ and $\left\{\sigma_{R}^{2}(t, \omega), t \geq 0\right\}$ as follows:

$$
\begin{gathered}
\mu_{R}(t, \omega)=\mathbb{E}\left[r\left(t, \omega, Y_{k}\right) \mid \mathcal{F}_{t-}\right]=\int_{0}^{M} r(t, \omega, y) \mathrm{d} F(y), \\
\sigma_{R}^{2}(t, \omega)=\mathbb{E}\left[r^{2}\left(t, \omega, Y_{k}\right) \mid \mathcal{F}_{t-}\right]=\int_{0}^{M} r^{2}(t, \omega, y) \mathrm{d} F(y) .
\end{gathered}
$$

Here $Y_{k}$, independent of $\mathcal{F}_{t-}$, has the distribution function $F$. Then, given an admissible control strategy $\pi=\left\{R_{t}, t \geq 0\right\}$, the controlled process $\left\{Z_{t}^{\pi}, t \geq 0\right\}$ with initial capital $x$ is the strong solution to the stochastic differential equation

$$
\mathrm{d} Z_{t}^{\pi}=\left[\delta\left(Z_{t}^{\pi}\right)+c+\xi \lambda \mu_{R}(t, \omega)-(1+\xi) \lambda \mu\right] \mathrm{d} t+\sqrt{\lambda} \sigma_{R}(t, \omega) \mathrm{d} B_{t}, \quad Z_{0}^{\pi}=x .
$$


As for the existence and uniqueness of the solution to a stochastic differential equation with random coefficients, we refer the reader to Fleming and Soner (1993, Chapter IV and Appendix D).

In the diffusion model (2.6), the absolute ruin time is defined as the first time when the drift coefficient turns negative. Denote by $\Pi$ the set of all admissible control policies. For every admissible strategy $\pi \in \Pi$, the absolute ruin time $\tau^{\pi}$ with respect to $Z_{t}^{\pi}$ is defined by

$$
\tau^{\pi}=\inf \left\{t \geq 0: \delta\left(Z_{t}^{\pi}\right)+c+\xi \lambda \mu_{R}(t, \omega)-(1+\xi) \lambda \mu<0\right\} .
$$

The insurer's objective is to minimize the probability of absolute ruin by dynamically adjusting the reinsurance position. The performance function associated with this control problem is defined as

$$
\Psi^{\pi}(x)=\mathbb{P}\left\{\tau^{\pi}<\infty \mid Z_{0}^{R}=x\right\}
$$

and the value function is defined as

$$
\Psi(x)=\inf _{\pi \in \Pi} \Psi^{\pi}(x) .
$$

In this paper our aim is to give the expression of the value function $\Psi(x)$ and find the optimal strategy $\pi^{*}=\left\{R_{t}^{*}\right\} \in \Pi$ such that $\Psi(x)=\Psi^{\pi^{*}}(x)$.

Note that the drift coefficient of $Z_{t}^{\pi}$ in (2.6) can be rewritten as

$$
\delta\left(Z_{t}^{\pi}\right)+c-\lambda \mu-\xi \lambda\left[\mu-\mu_{R}(t, \omega)\right] .
$$

Given the current surplus $Z_{t}^{\pi}=z$, if the insurer does not take reinsurance, i.e. $r(t, \omega, y)=y$ and $\mu_{R}(t, \omega)=\mu$, the drift coefficient of the controlled process becomes $\delta(z)+c-\lambda \mu$, which is the maximum value of the drift coefficients for all admissible reinsurance policies since $0 \leq \mu_{R}(t, \omega) \leq \mu$. Define a critical level $\tilde{d}$ by

$$
\tilde{d}=\sup \{z: \delta(z)+c-\lambda \mu \leq 0\}
$$

with $\tilde{d}=-\infty$ if the set is null. Note that $\delta(x)$ is a nondecreasing function and $0 \leq \mu_{R}(t, \omega) \leq$ $\mu$. Once the current surplus drops below $\tilde{d}$, we cannot find an admissible reinsurance to keep the drift coefficient positive. Thus, according to (2.7), the ultimate time of absolute ruin associated with the optimal strategy $\pi^{*}$ can be written as

$$
\tau^{\pi^{*}}=\inf \left\{t \geq 0: Z_{t}^{\pi^{*}}<\tilde{d}\right\}
$$

\section{The optimal form of reinsurance among a class of general reinsurance strategies}

In this section we will show that the excess-of-loss reinsurance policy is the optimal form of reinsurance for the insurer with performance function (2.8) and value function (2.9). In order to prove this result, we begin with some lemmas.

Lemma 3.1. (Gronwall inequality.) Suppose that $h(t)$ is a nonnegative continuous function satisfying

$$
h(t) \leq C+A \int_{0}^{t} h(s) \mathrm{d} s, \quad 0 \leq t \leq T,
$$

for some constants $C \geq 0$ and $A \geq 0$. Then

$$
h(t) \leq C \mathrm{e}^{A t}, \quad 0 \leq t \leq T .
$$


Proof. Lemma 3.1 can be obtained directly from Lemma 1 of Pachpatte (1973).

Lemma 3.2. Assume that there are two $\mathcal{F}_{t}$-predictable continuous processes $\left\{X_{t}^{i}\right\}, i=1,2$, satisfying the stochastic differential equations

$$
\mathrm{d} X_{t}^{i}=\left[\delta\left(X_{t}^{i}\right)+b_{i}(t, \omega)\right] \mathrm{d} t+\sigma(t, \omega) \mathrm{d} B_{t}, \quad X_{0}^{i}=x, i=1,2,
$$

where $\delta(x)$ satisfies (2.2) and (2.3), and $\left\{b_{1}(t, \omega)\right\},\left\{b_{2}(t, \omega)\right\}$, and $\{\sigma(t, \omega)\}$ are predictable càglàd processes. If

$$
b_{1}(t, w) \geq b_{2}(t, w) \quad \mathbb{P} \text {-almost surely }(\mathbb{P} \text {-a.s. }) \text { for every } t \geq 0
$$

then

$$
\mathbb{P}\left\{X_{t}^{1} \geq X_{t}^{2} \text { for every } 0 \leq t<\infty\right\}=1 .
$$

Proof. From (3.1) we know that $\left\{X_{t}^{i}\right\}, i=1,2$, satisfy the condition

$$
\mathrm{d}\left(X_{t}^{1}-X_{t}^{2}\right)=\left[\delta\left(X_{t}^{1}\right)-\delta\left(X_{t}^{2}\right)+b_{1}(t, \omega)-b_{2}(t, \omega)\right] \mathrm{d} t,
$$

which implies that the difference $X_{t}^{1}-X_{t}^{2}$ is continuous and differentiable with respect to $t$. Since $\left\{b_{i}(t, \omega)\right\}, i=1,2$, are left-continuous, by (3.2), we know that

$$
\mathbb{P}\left\{b_{1}(t, w) \geq b_{2}(t, w) \text { for every } 0 \leq t<\infty\right\}=1 .
$$

Let

$$
\Omega^{\prime}=\left\{\omega: b_{1}(t, w)>b_{2}(t, w) \text { for every } 0 \leq t<\infty\right\} .
$$

First we consider the case in which $\mathbb{P}\left\{\Omega^{\prime}\right\}=1$. Given $\omega \in \Omega^{\prime}$, if there exists $t>0$ such that $X_{t}^{1}(\omega)<X_{t}^{2}(\omega)$, because of the continuity property of $X_{t}^{i}(\omega), i=1,2$, we can find $t_{0} \geq 0$ and $\varepsilon>0$ such that $X_{t_{0}}^{1}(\omega)=X_{t_{0}}^{2}(\omega)$ and $X_{t}^{1}(\omega)<X_{t}^{2}(\omega)$ for every $t \in\left(t_{0}, t_{0}+\varepsilon\right)$. Hence, we have

$$
\begin{aligned}
\lim _{t \rightarrow t_{0}-} \frac{\mathrm{d}}{\mathrm{d} t}\left(X_{t}^{1}-X_{t}^{2}\right) & =\lim _{t \rightarrow t_{0}-}\left[\delta\left(X_{t}^{1}(\omega)\right)-\delta\left(X_{t}^{2}(\omega)\right)+b_{1}(t, \omega)-b_{2}(t, \omega)\right] \\
& =\delta\left(X_{t_{0}}^{1}(\omega)\right)-\delta\left(X_{t_{0}}^{2}(\omega)\right)+\lim _{t \rightarrow t_{0}-}\left[b_{1}(t, \omega)-b_{2}(t, \omega)\right] \\
& =b_{1}\left(t_{0}, \omega\right)-b_{2}\left(t_{0}, \omega\right) \\
& >0 .
\end{aligned}
$$

This contradicts $X_{t}^{1}(\omega)<X_{t}^{2}(\omega)$ for every $t \in\left(t_{0}, t_{0}+\varepsilon\right)$. Thus, we have

$$
\mathbb{P}\left\{X_{t}^{1} \geq X_{t}^{2} \text { for every } 0 \leq t<\infty\right\}=1 .
$$

Next we consider the case in which $\mathbb{P}\left\{\Omega^{\prime}\right\}<1$. In this case we construct a sequence of processes $\left\{X_{t}^{1, n}\right\}_{n=1}^{\infty}$ satisfying

$$
\mathrm{d} X_{t}^{1, n}=\left(\delta\left(X_{t}^{1, n}\right)+b_{1}(t, \omega)+\frac{1}{n}\right) \mathrm{d} t+\sigma(t, \omega) \mathrm{d} B_{t}, \quad X_{0}^{1, n}=x .
$$

According to the first case we know that $\left\{X_{t}^{1, n}\right\}_{n=1}^{\infty}$ is decreasing with $n$ and

$$
\mathbb{P}\left\{X_{t}^{1, n} \geq X_{t}^{i} \text { for every } t \geq 0\right\}=1, \quad i=1,2, n \geq 1 .
$$


In addition, for any fixed $T>0$ and $0 \leq t \leq T$, we have

$$
X_{t}^{1, n}-X_{t}^{1}=\int_{0}^{t}\left[\delta\left(X_{s}^{1, n}\right)-\delta\left(X_{s}^{1}\right)+\frac{1}{n}\right] \mathrm{d} s \leq L \int_{0}^{t}\left(X_{s}^{1, n}-X_{s}^{1}\right) \mathrm{d} s+\frac{T}{n} .
$$

Therefore, it follows from $X_{t}^{1, n}-X_{t}^{1} \geq 0$ a.s. and the Gronwall inequality that

$$
0 \leq X_{t}^{1, n}-X_{t}^{1} \leq \frac{T}{n} \mathrm{e}^{L t} \text { a.s. }
$$

Hence, for every $0 \leq t<\infty$, we have $X_{t}^{1, n} \searrow X_{t}^{1}$ a.s. as $n \rightarrow \infty$. Thus, $X_{t}^{1} \geq X_{t}^{2}$ a.s. and, thence, the desired result follows from the continuity of the processes $\left\{X_{t}^{i}\right\}, i=1,2$.

Lemma 3.3. For any given admissible policy $\pi=\left\{R_{t}\right\} \in \Pi$, there exists an admissible policy $\tilde{\pi}=\left\{\tilde{R}_{t}\right\} \in \Pi$, with $\tilde{R}_{t}=y \wedge m(t, \omega)$, such that

$$
\mathbb{P}\left\{Z_{t}^{\tilde{\pi}} \geq Z_{t}^{\pi} \text { for every } 0 \leq t<\infty\right\}=1 .
$$

Proof. Given $\pi=\left\{R_{t}:=r(t, \omega, y)\right\} \in \Pi$, it follows from $0 \leq r(t, \omega, y) \leq y$ that $0 \leq \sigma_{R}^{2}(t, \omega) \leq \sigma^{2}$. For every $m \in[0, M]$, we define

$$
\mu_{\mathrm{e}}(m)=\mathbb{E}\left(Y_{k} \wedge m\right)=\int_{0}^{m} \bar{F}(y) \mathrm{d} y, \quad \sigma_{\mathrm{e}}^{2}(m)=\mathbb{E}\left(Y_{k} \wedge m\right)^{2}=\int_{0}^{m} 2 y \bar{F}(y) \mathrm{d} y .
$$

It is easy to see that $0 \leq \sigma_{\mathrm{e}}^{2}(m) \leq \sigma^{2}$ and $\sigma_{\mathrm{e}}^{2}(m)$ is a strictly increasing function on $[0, M]$. Hence, there exists a unique $m(t, \omega) \in[0, M]$ such that $\sigma_{\mathrm{e}}^{2}(m(t, \omega))=\sigma_{R}^{2}(t, \omega)$, namely,

$$
m(t, \omega)=\sigma_{\mathrm{e}}^{2-1}\left(\sigma_{R}^{2}(t, \omega)\right),
$$

where $\sigma_{\mathrm{e}}^{2-1}(\cdot)$ denotes the inverse function of $\sigma_{\mathrm{e}}^{2}(\cdot)$.

Define an excess-of-loss reinsurance policy $\tilde{\pi}=\left\{\tilde{R}_{t}\right\}$ by

$$
\tilde{R}_{t}:=\tilde{r}(t, \omega, y)=y \wedge m(t, \omega) .
$$

Since $\sigma_{\mathrm{e}}^{2-1}(\cdot)$ is strictly increasing and continuous, and $\left\{\sigma_{R}^{2}(t, \omega), t \geq 0\right\}$ is predictable càglàd, $\left\{\tilde{R}_{t}:=\tilde{r}(t, \omega, y), t \geq 0\right\}$ is a $U$-valued predictable càglàd stochastic process with respect to $\left\{\mathcal{F}_{t}\right\}$ and it satisfies $0 \leq \tilde{r}(t, \omega, y) \leq y$. Hence, $\tilde{\pi}$ is an admissible reinsurance policy. In addition, we have $\sigma_{\tilde{R}}^{2}(t, \omega)=\sigma_{R}^{2}(t, \omega)$. In what follows we show that $\mu_{\tilde{R}}(t, \omega) \geq \mu_{R}(t, \omega)$. Note that

$$
\begin{aligned}
\sigma_{\tilde{R}}^{2}(t, \omega) & =\mathbb{E}\left[\left(\tilde{r}\left(t, \omega, Y_{k}\right)-m(t, \omega)\right)^{2} \mid \mathcal{F}_{t-}\right]+2 m(t, \omega) \mu_{\tilde{R}}(t, \omega)-m^{2}(t, \omega), \\
\sigma_{R}^{2}(t, \omega) & =\mathbb{E}\left[\left(r\left(t, \omega, Y_{k}\right)-m(t, \omega)\right)^{2} \mid \mathcal{F}_{t-}\right]+2 m(t, \omega) \mu_{R}(t, \omega)-m^{2}(t, \omega)
\end{aligned}
$$

and

$$
\begin{aligned}
\mathbb{E}\left[\left(r\left(t, \omega, Y_{k}\right)-m(t, \omega)\right)^{2} \mid \mathcal{F}_{t-}\right] \\
\quad=\int_{0}^{m(t, \omega)}[r(t, \omega, y)-m(t, \omega)]^{2} \mathrm{~d} F(y)+\int_{m(t, \omega)}^{M}[r(t, \omega, y)-m(t, \omega)]^{2} \mathrm{~d} F(y) \\
\quad \geq \int_{0}^{m(t, \omega)}[y-m(t, \omega)]^{2} \mathrm{~d} F(y) \\
\quad=\int_{0}^{m(t, \omega)}[y \wedge m(t, \omega)-m(t, \omega)]^{2} \mathrm{~d} F(y) \\
\quad=\mathbb{E}\left[\left(\tilde{r}\left(t, \omega, Y_{k}\right)-m(t, \omega)\right)^{2} \mid \mathcal{F}_{t-}\right] .
\end{aligned}
$$


Thus, it follows from (3.4), (3.5), and $\sigma_{\tilde{R}}^{2}(t, \omega)=\sigma_{R}^{2}(t, \omega)$ that

$$
\mu_{\tilde{R}}(t, \omega) \geq \mu_{R}(t, \omega) .
$$

By Lemma 3.2 and comparing the drift coefficients of the controlled stochastic processes $\left\{Z_{t}^{\pi}\right\}$ and $\left\{Z_{t}^{\tilde{\pi}}\right\}$, we have $\mathbb{P}\left\{Z_{t}^{\tilde{\pi}} \geq Z_{t}^{\pi}\right.$ for every $\left.t \geq 0\right\}=1$, which completes the proof of Lemma 3.3.

Now, we are ready to give the main result of this section, which states that a dynamic excess-of-loss reinsurance is the optimal form of reinsurance in the admissible reinsurance class $\Pi$.

Theorem 3.1. With the controlled surplus process described by (2.6) and the value function (2.9), the optimal reinsurance policy among the class of admissible reinsurance policies $\Pi$ is in the form of excess-of-loss reinsurance.

Proof. Given any admissible reinsurance policy $\pi$, we take the same admissible excessof-loss reinsurance policy $\tilde{\pi}$ described in Lemma 3.3. Also note that $\delta(x)$ is a nondecreasing function. By the result $\mathbb{P}\left\{Z_{t}^{\tilde{\pi}} \geq Z_{t}^{\pi}\right.$ for every $\left.0 \leq t<\infty\right\}=1$, (3.6), and (2.7), we have $\tau^{\pi} \leq$ $\tau^{\tilde{\pi}}$, which implies that $\Psi^{\tilde{\pi}}(x) \leq \Psi^{\pi}(x)$. In other words, for any admissible reinsurance policy $\pi$, we can find an admissible excess-of-loss reinsurance such that the absolute ruin probability under the excess-of-loss reinsurance is less than or equal to the absolute ruin probability under the policy $\pi$. Hence, the optimal reinsurance policy is in the form of excess-of-loss reinsurance.

Remark 3.1. Note that the classical ruin is a special case of the absolute ruin; it is easy to see that, under the same assumptions used for Theorem 3.1, the excess-of-loss reinsurance is still the optimal form of reinsurance if we take the minimizing ruin probability as the optimization criterion.

Remark 3.2. In Theorem 3.1 we proved the optimality of the excess-of-loss reinsurance under the expected value premium principle and the criterion of minimizing the absolute ruin probability. However, we point out that under different premium principles the optimal reinsurance forms would be different; see, e.g. Zhou and Yuen (2012). Furthermore, the technique used in Section 3 of our paper can be used to study optimal dynamic risk control problems under other optimization criteria such as maximizing the total expected dividends paid up to ruin time and maximizing the expected utility of the insurer's wealth at a fixed time.

\section{The explicit solution for the optimal dynamic excess-of-loss reinsurance}

In this section we illustrate the application of the results of Section 3 by deriving the explicit solutions to the value function and the optimal reinsurance. Since the optimal reinsurance policy is in the form of excess-of-loss reinsurance, the problem of obtaining the optimal reinsurance policy is reduced to the problem of determining the optimal retention levels among the class of excess-of-loss reinsurance strategies. In doing so we redefine the control policies and the controlled stochastic process among the class of excess-of-loss reinsurance strategies.

First, the set of all admissible policies, denoted by $\Pi_{e}$, is defined as

$$
\Pi_{\mathrm{e}}=\left\{\left\{m_{t}, t \geq 0\right\}:\left\{m_{t}\right\} \text { is a } \mathcal{F}_{t} \text {-predictable càglàd process with } 0 \leq m_{t} \leq M\right\} .
$$

Given an admissible control policy $\pi_{\mathrm{e}}=\left\{m_{t}, t \geq 0\right\} \in \Pi_{\mathrm{e}}$, let $\left\{Z_{t}^{\pi_{\mathrm{e}}}\right\}$ denote the surplus process under the excess-of-loss reinsurance $\pi_{\mathrm{e}}$ with dynamic retention level $\left\{m_{t}\right\}$. Then, the controlled 
surplus process can be rewritten as

$$
\mathrm{d} Z_{t}^{\pi_{\mathrm{e}}}=\left[\delta\left(Z_{t}^{\pi_{\mathrm{e}}}\right)+c+\xi \lambda \mu_{\mathrm{e}}\left(m_{t}\right)-(1+\xi) \lambda \mu\right] \mathrm{d} t+\sqrt{\lambda} \sigma_{\mathrm{e}}\left(m_{t}\right) \mathrm{d} B_{t}, \quad Z_{0}^{\pi_{\mathrm{e}}}=x,
$$

where $\mu_{\mathrm{e}}(\cdot)$ and $\sigma_{\mathrm{e}}^{2}(\cdot)$ are defined by (3.3). Correspondingly, the time of absolute ruin is

$$
\tau^{\pi_{\mathrm{e}}}=\inf \left\{t \geq 0: \delta\left(Z_{t}^{\pi_{\mathrm{e}}}\right)+c+\xi \lambda \mu_{\mathrm{e}}\left(m_{t}\right)-(1+\xi) \lambda \mu<0\right\}
$$

and the value function is

$$
\Psi(x)=\inf _{\pi_{\mathrm{e}} \in \Pi_{\mathrm{e}}} \Psi^{\pi_{\mathrm{e}}}(x)=\inf _{\pi_{\mathrm{e}} \in \Pi_{\mathrm{e}}} \mathbb{P}\left\{\tau^{\pi_{\mathrm{e}}}<\infty \mid Z_{0}^{\pi_{\mathrm{e}}}=x\right\} .
$$

In this section we will find the expressions of the value function and the optimal retention level $\pi_{\mathrm{e}}^{*}=\left\{m_{t}^{*}, t \geq 0\right\} \in \Pi_{\mathrm{e}}$ such that $\Psi(x)=\Psi^{\pi_{\mathrm{e}}^{*}}(x)$.

Before solving this optimal control problem we first analyze the boundary conditions satisfied by the value function $\Psi(x)$. Recall the critical level $\tilde{d}$ defined in (2.10) and note that, by the definition (and probability) of absolute ruin, we know that $\Psi(x)=1$ for $x \leq \tilde{d}$ because, for any admissible policy $\pi_{\mathrm{e}}=\left\{m_{t}\right\} \in \Pi_{\mathrm{e}}$, we have

$$
\delta(x)+c+\xi \lambda \mu_{\mathrm{e}}\left(m_{t}\right)-(1+\xi) \lambda \mu \leq \delta(x)+c-\lambda \mu \leq 0,
$$

which implies that $\Psi^{\pi_{\mathrm{e}}}(x)=1$.

For $x>\tilde{d}$, by taking the special policy $\pi_{\mathrm{e}}^{M}=\left\{m_{t}=M, t \geq 0\right\}$ and the results of Gerber and Yang (2007), we have $\Psi^{\pi_{\mathrm{e}}^{M}}(x)<1$, which implies that $\Psi(x)<1$ for $x>\tilde{d}$.

In addition, we define another critical level $\bar{x}$ by

$$
\bar{x}=\sup \{x: \delta(x)+c-\lambda \mu<\xi \lambda \mu\}
$$

with $\bar{x}=\tilde{d}$ if the set is null. It is easy to see that $\bar{x} \geq \tilde{d}$. For $x \geq \bar{x}$, if we take the special policy

$$
\pi_{\mathrm{e}}^{0}=\left\{m_{t}=0, t \geq 0\right\}
$$

then the drift coefficient of the controlled process $\left\{Z_{t}^{\pi_{\mathrm{e}}^{0}}\right\}$ is nonnegative while the diffusion coefficient is 0 . Thus, in this case, we have $\Psi^{\pi_{\mathrm{e}}^{0}}(x)=0$, which implies that $\Psi(x)=0$ for $x \geq \bar{x}$. In particular, if $\bar{x}=\tilde{d}$ then $\Psi(x)=0$ for $x \geq \tilde{d}$. Therefore, we assume that $\bar{x}>\tilde{d}$ in the rest of the paper.

In summary, the value function satisfies

$$
\begin{aligned}
\Psi(x) & =0, & & \bar{x} \leq x, \\
0<\Psi(x) & <1, & & \tilde{d}<x<\bar{x}, \\
\Psi(x) & =1, & & x \leq \tilde{d} .
\end{aligned}
$$

In what follows we will give the explicit solutions to the value function and the optimal retention level. To this end, we first construct a candidate solution to the HJB equation and find the optimal retention level. Then we show that the candidate solution is just the value function in a verification theorem.

Suppose that the value function $\Psi(x)$ is twice continuously differentiable on $(\tilde{d}, \bar{x})$. Employing the standard theory in Fleming and Soner (1993), $\Psi(x)$ satisfies the HJB equation

$$
\inf _{m \in[0, M]} \frac{1}{2} \lambda \sigma_{\mathrm{e}}^{2}(m) \Psi_{x x}(x)+\left[\delta(x)+c-\lambda \mu-\xi \lambda\left(\mu-\mu_{\mathrm{e}}(m)\right)\right] \Psi_{x}(x)=0, \quad \tilde{d}<x<\bar{x},
$$

with boundary conditions $\Psi(\tilde{d})=1$ and $\Psi(\bar{x})=0$. 
Now, differentiating with respect to $m$ on both sides of (4.3), we obtain

$$
\lambda \bar{F}(m)\left[m \Psi_{x x}(x)+\xi \Psi_{x}(x)\right]=0
$$

which implies that the maximizer satisfies the following three cases.

Case (i). We have

$$
m(x)=-\xi \frac{\Psi_{x}(x)}{\Psi_{x x}(x)}, \quad \Psi_{x x}(x) \neq 0, \quad \text { and } \quad 0<m(x)<M .
$$

Case (ii). $\bar{F}(m)=0$, which implies that $m=M$.

Case (iii). $\Psi_{x}(x)=0$ and $\Psi_{x x}(x)=0$.

Let us first consider case (i). By combining (4.4) with (4.3), we obtain

$$
g(x, m) \Psi_{x x}(x)=0,
$$

where

$$
g(x, m)=\frac{1}{2} \xi \lambda \sigma_{\mathrm{e}}^{2}(m)-m\left[\delta(x)+c-\lambda \mu-\xi \lambda\left(\mu-\mu_{\mathrm{e}}(m)\right)\right] .
$$

Therefore, we need to find $0<m(x)<M$ such that $g(x, m)=0$. To do this, we have the following lemma concerning the solution to the equation $g(x, m)=0$.

Note that $M$ could be either finite and infinite. In the following lemma, if $M$ appears in the form of $1 / M$, we mean $1 / M=0$ when $M$ is $\infty$.

Lemma 4.1. Recall the critical levels $\tilde{d}$ and $\bar{x}$ in (2.10) and (4.1). Define

$$
\underline{x}=\inf \left\{x: \delta(x)+c-\lambda \mu>\xi \lambda \frac{\sigma^{2} / 2}{M}\right\} .
$$

If $x \in[\bar{x}, \infty)$ then $g(x, m)<0$ for all $m>0$. If $x \in(\tilde{d}, \bar{x})$ then there exists $\bar{m}(x)>0$ such that $g(x, \bar{m})=0$. In particular,

1. if $x \in(\tilde{d}, \underline{x}]$ then $\bar{m}(x) \geq M$,

2. if $x \in(\underline{x}, \bar{x})$ then $0<\bar{m}(x)<M$,

3. $\bar{m}(\bar{x})=0$ and $\bar{m}(\underline{x})=M$.

Proof. We first need to show that $\tilde{d} \leq \underline{x}<\bar{x}$. Because $\delta(x)$ is increasing, it is easy to see that $\tilde{d} \leq \underline{x}$. From (4.1) and (4.5), the inequality $\underline{x}<\bar{x}$ follows from

$$
\xi \lambda \frac{\sigma^{2} / 2}{M}=\xi \lambda \frac{\left(\int_{0}^{M} 2 x \bar{F}(x) \mathrm{d} x\right) / 2}{M}<\xi \lambda \int_{0}^{M} \bar{F}(x) \mathrm{d} x=\xi \lambda \mu .
$$

For any given $x>\tilde{d}$, it is easy to verify that $g(x, 0)=0, \lim _{m \rightarrow \infty} g(x, m)=-\infty$, and

$$
\begin{gathered}
\frac{\partial}{\partial m} g(x, m)=-\left[\delta(x)+c-\lambda \mu-\xi \lambda\left(\mu-\mu_{\mathrm{e}}(m)\right)\right], \\
\frac{\partial^{2}}{\partial m^{2}} g(x, m)=-\xi \lambda \bar{F}(m) .
\end{gathered}
$$


If $m \geq M$ then

$$
\frac{\partial}{\partial m} g(x, m)=-[\delta(x)+c-\lambda \mu]<0, \quad \frac{\partial^{2}}{\partial m^{2}} g(x, m)=0 .
$$

If $m<M$ then

$$
\frac{\partial^{2}}{\partial m^{2}} g(x, m)<0 .
$$

Thus, for any given $x>\tilde{d}$, the function $g(x, m)$ is strictly concave with respect to $m$ on $[0, M]$ and linearly decreasing on $(M, \infty)$.

If

$$
\left.\frac{\partial}{\partial m} g(x, m)\right|_{m=0}=-[\delta(x)+c-(1+\xi) \lambda \mu]>0
$$

holds, namely, when $x<\bar{x}$, then we can see that the equation $g(x, m)=0$ has one positive root, say $\bar{m}(x)$. Otherwise, if $x>\bar{x}$, we have $g(x, m)<g(x, 0)=0$ for all $m>0$. For $x=\bar{x}$, we have

and so $\bar{m}(\bar{x})=0$.

$$
\left.\frac{\partial}{\partial m} g(x, m)\right|_{m=0}=0 \text {, }
$$

For $\tilde{d}<x<\bar{x}$, if $g(x, M)<0$, namely, when $x \in(\underline{x}, \bar{x})$, then we have $0<\bar{m}(x)<M$. Otherwise, if $x \in(\tilde{d}, \underline{x}), \bar{m}(x)>M$. For $x=\underline{x}$, we have $g(\underline{x}, M)=0$, which implies that $\bar{m}(\underline{x})=M$. This completes the proof.

In the following proposition we study the property of the solution $\bar{m}(x)$ for $\tilde{d}<x<\bar{x}$, which shows that the solution $\bar{m}(x)$ is decreasing, and the left derivative of $\bar{m}(x)$ at $x=\bar{x}$ is proportional to the derivative of the function $\delta(x)$.

Proposition 4.1. For any $\tilde{d}<x<\bar{x}$, if $\delta(x)$ is differentiable then $\bar{m}^{\prime}(x) \leq 0$ and

$$
\lim _{x \rightarrow \bar{x}-} \bar{m}^{\prime}(x)=-\frac{2}{\xi \lambda} \delta^{\prime}(\bar{x}-) .
$$

Proof. Note that $\bar{m}(x)$ satisfies the equation $g(x, \bar{m}(x))=0$. Dividing both sides of this equation by $\bar{m}(x)$, we have

$$
\xi \lambda \frac{\sigma_{\mathrm{e}}^{2}(\bar{m}(x)) / 2}{\bar{m}(x)}=\delta(x)+c-\lambda \mu-\xi \lambda\left[\mu-\mu_{\mathrm{e}}(\bar{m}(x))\right] .
$$

Taking the derivative of both sides and then simplifying the expression, we obtain

$$
-\xi \lambda \bar{m}^{\prime}(x) \frac{\sigma_{\mathrm{e}}^{2}(\bar{m}(x)) / 2}{\bar{m}^{2}(x)}=\delta^{\prime}(x) .
$$

Thus, $\bar{m}^{\prime}(x) \leq 0$ since $\delta(x)$ is nondecreasing. Noting that $\bar{m}(x) \rightarrow 0$ as $x \rightarrow \bar{x}-$, we have

$$
\lim _{x \rightarrow \bar{x}-} \frac{\sigma_{\mathrm{e}}^{2}(\bar{m}(x))}{\bar{m}^{2}(x)}=\lim _{m \rightarrow 0+} \frac{\int_{0}^{m} 2 y \bar{F}(y) \mathrm{d} y}{m^{2}}=1 .
$$

Thus,

$$
\lim _{x \rightarrow \bar{x}-} m^{\prime}(x)=\lim _{x \rightarrow \bar{x}-}-\frac{2 \delta^{\prime}(x)}{\xi \lambda} \frac{\bar{m}^{2}(x)}{\sigma_{\mathrm{e}}^{2}(\bar{m}(x))}=-\frac{2}{\xi \lambda} \delta^{\prime}(\bar{x}-),
$$

which completes the proof. 
By Lemma 4.1, we know that, for $\underline{x}<x<\bar{x}$, there exists $m(x)=\bar{m}(x) \in(0, M)$ such that (4.4) holds. For case (ii) and case (iii), we take $m(x)=M$ for $x \leq \underline{x}$ and $m(x)=0$ for $x \geq \bar{x}$, respectively. Define the maximizer of the retention level in (4.3) as

$$
m(x)= \begin{cases}0, & \bar{x} \leq x, \\ \bar{m}(x), & \underline{x}<x<\bar{x}, \\ M, & \tilde{d}<x \leq \underline{x} .\end{cases}
$$

With this maximizer $m(x)$ as a candidate of the optimal retention level, we can construct the candidate solution of (4.3) as follows.

When $x \geq \bar{x}$, taking $m(x)=0$ in (4.3) gives $\Psi^{\prime}(x)=0$, and, hence, $\Psi^{\prime \prime}(x)=0$, which is coincident to case (iii). By the boundary condition $\Psi(\bar{x})=0$ in (4.2), we have $\Psi(x)=0$ for $x \geq \bar{x}$

When $\underline{x}<x<\bar{x}$ and $\tilde{d}<x \leq \underline{x}$, combining $m(x)=\bar{m}(x)$ with (4.4) and inserting $m(x)=M$ into (4.3), we obtain

$$
\begin{array}{cc}
\Psi(x)=k_{2} \int_{\underline{x}}^{x} \exp \{-\xi G(t)\} \mathrm{d} t+k_{1}, & \underline{x}<x<\bar{x}, \\
\Psi(x)=l_{2} \int_{\tilde{d}}^{x} \exp \left\{-\frac{2}{\sigma^{2}} H(t)\right\} \mathrm{d} t+l_{1}, & \tilde{d}<x \leq \underline{x},
\end{array}
$$

where

$$
\begin{gathered}
G(t)=\int_{\underline{x}}^{t} \bar{m}^{-1}(s) \mathrm{d} s, \\
H(t)=\frac{1}{\lambda} \int_{\tilde{d}}^{t} \delta(s) \mathrm{d} s+\left(\frac{c}{\lambda}-\mu\right) t .
\end{gathered}
$$

By the boundary conditions and the smooth conditions, we have the four equations

$$
\begin{array}{cc}
\Psi(\bar{x})=0, & \Psi(\underline{x}-)=\Psi(\underline{x}+), \\
\Psi(\tilde{d})=1, & \Psi^{\prime}(\underline{x}-)=\Psi^{\prime}(\underline{x}-),
\end{array}
$$

which are used to determine the four constants $k_{i}$ and $l_{i}$ for $i=1,2$ in (4.7) and (4.8). Thus, we give a candidate solution, say $\gamma(x)$, to the value function $\Psi(x)$, where

$$
\gamma(x)= \begin{cases}0, & \bar{x} \leq x, \\ k_{2} \int_{\underline{x}}^{x} \exp \{-\xi G(t)\} \mathrm{d} t+k_{1}, & \underline{x}<x<\bar{x}, \\ l_{2} \int_{\tilde{d}}^{x} \exp \left\{-\frac{2}{\sigma^{2}} H(t)\right\} \mathrm{d} t+l_{1}, & \tilde{d}<x \leq \underline{x} .\end{cases}
$$

We have the following proposition about the function $\gamma(x)$.

Proposition 4.2. The function $\gamma(x)$ in (4.10) is differentiable for every $x>\tilde{d}$ and it is twice differentiable on $(\tilde{d}, \bar{x})$. 
Proof. We just need to verify the twice differentiable property of $\gamma(x)$ at $x=\underline{x}$ and differentiability at $x=\bar{x}$. For $x>\underline{x}$,

$$
\gamma^{\prime \prime}(x)=-k_{2} \exp \{-\xi G(x)\} \xi G^{\prime}(x)=-\gamma^{\prime}(x) \frac{\xi}{\bar{m}^{-1}(x)},
$$

and, for $x<\underline{x}$,

$$
\gamma^{\prime \prime}(x)=-l_{2} \exp \left\{-\frac{2}{\sigma^{2}} H(t)\right\} \frac{2}{\sigma^{2}} H^{\prime}(x)=-\gamma^{\prime}(x) \frac{2}{\lambda \sigma^{2}}[\delta(x)+c-\lambda \mu] .
$$

Hence, by noting that $\bar{m}(\underline{x})=M$, we obtain

$$
\gamma^{\prime \prime}(\underline{x}-)=-\gamma^{\prime}(\underline{x}-)\left[\frac{2}{\lambda \sigma^{2}}(\delta(\underline{x})+c-\lambda \mu)\right]=-\gamma^{\prime}(\underline{x}-) \frac{\xi}{M}=-\gamma^{\prime}(\underline{x}+) \frac{\xi}{\bar{m}(\underline{x})}=\gamma^{\prime \prime}(\underline{x}+) \text {. }
$$

In addition, by noting that $\bar{m}(\bar{x})=0$, we have

$$
\gamma^{\prime}(\bar{x}-)=k_{2} H(\bar{x}-)=0=\gamma^{\prime}(\bar{x}+)
$$

completing the proof.

By summarizing the above discussions and Proposition 4.2, we have the following lemma.

Lemma 4.2. The function $\gamma(x)$ in (4.10) is a decreasing convex solution to (4.3) and the maximizer $m(x)$ is defined in (4.6).

In Lemma 4.2 we have a candidate solution $\gamma(x)$ for the value function $\Psi(x)$ and a maximizer $m(x)$ as a candidate to the optimal retention level. In what follows we will give the verification theorem which shows that the candidate solution and the maximizer are exactly the value function and the optimal policy.

Theorem 4.1. The value function $\Psi(x)=\gamma(x)$ and the optimal policy $\pi_{\mathrm{e}}^{*} \in \Pi_{\mathrm{e}}$ with $\pi_{\mathrm{e}}^{*}=$ $\left\{m_{t}^{*}, t \geq 0\right\}$ is defined by

$$
m_{t}^{*}= \begin{cases}0, & Z_{t}^{\pi_{\mathrm{e}}^{*}} \geq \bar{x}, \\ \bar{m}\left(Z_{t}^{\pi_{\mathrm{e}}^{*}}\right), & \underline{x}<Z_{t}^{\pi_{\mathrm{e}}^{*}}<\bar{x}, \\ M, & \overline{\tilde{d}}<Z_{t}^{\pi_{\mathrm{e}}^{*}} \leq \underline{x}\end{cases}
$$

Proof. By the definition of $\left\{m_{t}^{*}\right\}$ in (4.11) and Lemma 4.1, we know that $\left\{m_{t}^{*}\right\}$ is a predictable continuous stochastic process, so $\pi_{\mathrm{e}}^{*} \in \Pi_{\mathrm{e}}$. Meanwhile, the function $\gamma(x)$ is the probability of absolute ruin with control policy $\pi_{\mathrm{e}}^{*}$. Thus, $\gamma(x) \geq \Psi(x)$ by the definition of value function.

On the other hand, we need to show that $\gamma(x) \leq \Psi(x)$. Consider any control policy $\pi_{\mathrm{e}}=$ $\left\{m_{t}, t \geq 0\right\} \in \Pi_{\mathrm{e}}$. Define the first hitting time

$$
\tau_{\bar{x}}^{\pi_{\mathrm{e}}}=\inf \left\{t \geq 0: Z_{t}^{\pi_{\mathrm{e}}}=\bar{x}\right\} \quad(=\infty \text { if the set is null }) .
$$

Now we take a special admissible control policy $\tilde{\pi}_{\mathrm{e}}=\left\{\tilde{m}_{t}, t \geq 0\right\}$ of the form

$$
\tilde{m}_{t}= \begin{cases}m_{t}, & t<\tau_{\bar{x}}^{\pi_{\mathrm{e}}} \\ 0, & t \geq \tau_{\bar{x}}^{\pi_{\mathrm{e}}}\end{cases}
$$


Thus, we have $Z_{t}^{\pi_{\mathrm{e}}}=Z_{t}^{\tilde{\pi}_{\mathrm{e}}}$ for all $t \leq \tau_{\bar{x}}^{\pi_{\mathrm{e}}}$ and $\tau_{\bar{x}}^{\pi_{\mathrm{e}}}=\tau_{\bar{x}}^{\tilde{\pi}_{\mathrm{e}}}$. Since $\gamma(x)$ is twice differentiable on $(d, \bar{x})$ and satisfies (4.3) according to Proposition 4.2 and Lemma 4.2, we have, by Itô's formula,

$$
\begin{aligned}
& \gamma\left(Z_{\tau^{\tilde{\pi}} \tilde{\mathrm{e}}_{\mathrm{e}} \wedge \tau_{\tilde{x}}^{\tilde{\pi} \mathrm{e}} \wedge t}\right)=\gamma(x)+\lambda \int_{0}^{\tau^{\tilde{\tilde{\pi}}} \wedge \tau_{\bar{x}}^{\tilde{\pi} \mathrm{e}} \wedge t} \sigma^{2}\left(\tilde{m}_{S}\right) \gamma^{\prime}\left(Z_{s}^{\tilde{\pi}_{\mathrm{e}}}\right) \mathrm{d} B_{S} \\
& +\int_{0}^{\tau^{\tilde{\pi_{\mathrm{e}}}} \wedge \tau_{\overline{\tilde{x}}}^{\tilde{\tilde{\mathrm{e}}}} \wedge t} \frac{1}{2} \sigma^{2}\left(\tilde{m}_{S}\right) \gamma^{\prime \prime}\left(Z_{s}^{\tilde{\pi}_{\mathrm{e}}}\right) \mathrm{d} s \\
& +\int_{0}^{\tau^{\tilde{\pi_{\mathrm{e}}}} \wedge \tau_{\bar{x}}^{\tilde{\tilde{\pi}} \mathrm{e}} \wedge t}\left[\delta\left(Z_{s-}^{\tilde{\pi}_{\mathrm{e}}}\right)+c-\lambda \mu-\xi \lambda\left(\mu-\mu\left(\tilde{m}_{s}\right)\right)\right] \gamma^{\prime}\left(Z_{s}^{\tilde{\pi}_{\mathrm{e}}}\right) \mathrm{d} s \\
& \geq \gamma(x)+\lambda \int_{0}^{\tau^{\tilde{\tilde{e}_{\mathrm{e}}}} \wedge \tau_{\tilde{\bar{x}}}^{\tilde{\tilde{\mathrm{e}}_{\mathrm{e}}}} \wedge t} \sigma^{2}\left(\tilde{m}_{s}\right) \gamma^{\prime}\left(Z_{s}^{\tilde{\pi}_{\mathrm{e}}}\right) \mathrm{d} B_{s} .
\end{aligned}
$$

It follows from (4.10) that

$$
\left|\gamma^{\prime}(x)\right| \leq \max \left\{\left|k_{2}\right|,\left|l_{2}\right|\right\} \quad \text { for } \tilde{d}<x<\bar{x} .
$$

Thus, $\left|\sigma^{2}\left(\tilde{m}_{s}\right) \gamma^{\prime}\left(Z_{s}^{\tilde{\pi}_{\mathrm{e}}}\right)\right|$ is bounded by $\sigma^{2} \max \left\{\left|k_{2}\right|,\left|l_{2}\right|\right\}$ for $0 \leq s \leq \tau^{\tilde{\pi}_{\mathrm{e}}} \wedge \tau_{\overline{\bar{x}}}^{\tilde{\pi}_{\mathrm{e}}} \wedge t$. Hence, the stochastic integral on the right-hand side of (4.12) is a martingale. Taking the expectation of both sides of (4.12), we have

$$
\mathbb{E}\left[\gamma\left(Z_{\tau}^{\tau_{\tau_{\mathrm{e}} \wedge \tau_{\bar{x}}}^{\tilde{\pi}_{\mathrm{e}} \wedge t}}\right) \mid Z_{0}^{\tilde{\pi}_{\mathrm{e}}}=x\right] \geq \gamma(x), \quad \tilde{d}<x<\bar{x} .
$$

Moreover,

$$
\begin{aligned}
& \mathbb{E}\left[\gamma\left(Z_{\tau^{\tilde{\pi}_{\mathrm{e}}} \wedge \tau_{\bar{x}}^{\tilde{\pi}_{\mathrm{e}}} \wedge t}^{\tilde{\pi}_{\mathrm{e}}}\right) \mid Z_{0}^{\tilde{\pi}_{\mathrm{e}}}=x\right] \\
&= \gamma(\tilde{d}) \mathbb{P}\left\{\tau^{\tilde{\pi}_{\mathrm{e}}}<\tau_{\bar{x}}^{\tilde{\pi}_{\mathrm{e}}} \wedge t \mid Z_{0}^{\tilde{\pi}_{\mathrm{e}}}=x\right\}+\gamma(\bar{x}) \mathbb{P}\left\{\tau_{\bar{x}}^{\tilde{\pi}_{\mathrm{e}}}<\tau^{\tilde{\pi}_{\mathrm{e}}} \wedge t \mid Z_{0}^{\tilde{\pi}_{\mathrm{e}}}=x\right\} \\
&+\mathbb{E}\left[\gamma\left(Z_{t}^{\tilde{\pi}_{\mathrm{e}}}\right) I\left\{t<\tau_{\bar{x}}^{\tilde{\pi}_{\mathrm{e}}} \wedge \tau^{\tilde{\pi}_{\mathrm{e}}}\right\} \mid Z_{0}^{\tilde{\pi}_{\mathrm{e}}}=x\right] \\
&= \gamma(\tilde{d}) \mathbb{P}\left\{\tau^{\pi_{\mathrm{e}}}<\tau_{\bar{x}}^{\pi_{\mathrm{e}}} \wedge t \mid Z_{0}^{\pi_{\mathrm{e}}}=x\right\}+\mathbb{E}\left[\gamma\left(Z_{t}^{\tilde{\pi}_{\mathrm{e}}}\right) I\left\{t<\tau_{\bar{x}}^{\tilde{\pi}_{\mathrm{e}}} \wedge \tau^{\tilde{\pi}_{\mathrm{e}}}\right\} \mid Z_{0}^{\tilde{\pi}_{\mathrm{e}}}=x\right] \\
& \leq \gamma(\tilde{d}) \mathbb{P}\left\{\tau^{\pi_{\mathrm{e}}}<t \mid Z_{0}^{\pi_{\mathrm{e}}}=x\right\}+\mathbb{E}\left[\gamma\left(Z_{t}^{\tilde{\pi}_{\mathrm{e}}}\right) I\left\{t<\tau_{\bar{x}}^{\tilde{\pi}_{\mathrm{e}}} \wedge \tau^{\tilde{\pi}_{\mathrm{e}}}<\infty\right\} \mid Z_{0}^{\tilde{\pi}_{\mathrm{e}}}=x\right] \\
&+\mathbb{E}\left[\gamma\left(Z_{t}^{\tilde{\pi}_{\mathrm{e}}}\right) I\left\{\tau_{\bar{x}}^{\tilde{\pi}_{\mathrm{e}}} \wedge \tau^{\tilde{\pi}_{\mathrm{e}}}=\infty\right\} \mid Z_{0}^{\tilde{\pi}_{\mathrm{e}}}=x\right] .
\end{aligned}
$$

Note that there is a positive drift for $Z_{t}^{\tilde{\pi}_{\mathrm{e}}}$ on $\left\{\tau^{\tilde{\pi}_{\mathrm{e}}} \wedge \tau_{\bar{x}}^{\tilde{\pi}_{\mathrm{e}}}=\infty\right\}$ and, therefore, $\lim _{t \rightarrow \infty} Z_{t}^{\tilde{\pi}_{\mathrm{e}}}=\bar{x}$ for $\omega \in\left\{\tau^{\tilde{\pi}_{\mathrm{e}}} \wedge \tau_{\bar{x}}^{\tilde{\pi}_{\mathrm{e}}}=\infty\right\}$. Thus, letting $t \rightarrow+\infty$ on both sides of the above equation and using $\gamma(\bar{x})=0$, we obtain, by the dominated convergence theorem,

$$
\Psi^{\pi_{\mathrm{e}}}(x) \geq \frac{\gamma(x)}{\gamma(\tilde{d})}=\gamma(x), \quad \tilde{d}<x<\bar{x} .
$$

For $x \geq \bar{x}$, we have $\Psi^{\pi_{\mathrm{e}}}(x) \geq \gamma(x)=0$. Taking the infimum with respect to $\pi_{\mathrm{e}} \in \Pi_{\mathrm{e}}$, we obtain $\bar{\Psi}(x) \geq \gamma(x)$ for $x \geq \tilde{d}$, which completes the proof.

Remark 4.1. According to the optimal reinsurance form obtained in Theorem 4.1, we see that, when the surplus exceeds the level $\bar{x}$, the optimal retention level will be 0 . Meanwhile the 
probability of absolute ruin will be 0 , which means that the insurer can stay in a safe position by ceding all the risk exposures. In fact, when the surplus is so large that the investment income rate exceeds the cost of reinsuring all risk exposures, i.e.

$$
\delta(x) \geq(1+\xi) \lambda \mu-c \text { or } \quad x \geq \bar{x},
$$

the insurer could cede all the risks without risk of absolute ruin. From this point of view, we can conclude that it is somehow conservative to minimize the absolute ruin probability (or ruin probability), in the risk model with investment, for insurance portfolios with large surplus.

\section{Numerical examples and interpretation}

In this section we present two numerical examples to illustrate the results of Section 4 by considering the special risk model (2.4). Throughout this section, the parameter values are $c=1.2, \lambda=1, \mu=1, \Delta=5$, and $\xi=0.5$.

If the insurer does not take any reinsurance and retains all the risk, i.e. when $\pi_{\mathrm{e}}^{M}=$ $\left\{m_{t}=M, t \geq 0\right\}$, then the probability of absolute ruin without reinsurance in the diffusion approximation risk model satisfies

$$
\frac{1}{2} \lambda \sigma^{2} \Psi_{x x}^{\pi_{\mathrm{e}}^{M}}(x)+[\delta(x)+c-\lambda \mu] \Psi_{x}^{\pi_{\mathrm{e}}^{M}}(x)=0
$$

with boundary conditions $\Psi^{\pi_{\mathrm{e}}^{M}}(\tilde{d})=1$ and $\Psi^{\pi_{\mathrm{e}}^{M}}(\infty)=0$. The solution $\Psi^{\pi_{\mathrm{e}}^{M}}(x)$ can be expressed, by some elementary calculations, as

$$
\Psi^{\pi_{\mathrm{e}}^{M}}(x)=1-\frac{\int_{\tilde{d}}^{x} \exp \left\{-\left(2 / \sigma^{2}\right) H(t)\right\} \mathrm{d} t}{\int_{\tilde{d}}^{\infty} \exp \left\{-\left(2 / \sigma^{2}\right) H(t)\right\} \mathrm{d} t}, \quad x \geq \tilde{d},
$$

where the function $H(t)$ is defined in (4.9). For more detailed discussions about the solution of the probability of absolute ruin in the diffusion model, see Gerber and Yang (2007).

Example 5.1. (Exponential and Pareto claim distributions.) In this example we suppose that the claim sizes follow an exponential distribution and a Pareto distribution with the same mean value $\mu=1$. For a $\operatorname{Pareto}(\alpha, \theta)$ distribution, we have

$$
F(x)=1-\left(\frac{\theta}{x+\theta}\right)^{\alpha}, \quad x>0 .
$$

Here we take $\alpha=3$ and $\theta=2$. In the two cases, $M=\infty$. In Figure 1 we present the optimal retention levels for $x<0$ and $x \geq 0$. In Figure 2 we present the probabilities of absolute ruin with the optimal reinsurance and the probabilities of absolute ruin without reinsurance (with reference to (2.4)) for $\alpha=5 \%$ and $\beta=5 \%, 10 \%, 20 \%, \infty$, respectively. Note that the probability of absolute ruin is reduced to the probability of ruin when $\beta=\infty$.

Example 5.2. (Uniform claim distribution.) In this example we suppose that the claim sizes are uniformly distributed on [0,2]. In this case, $M=2<\infty$. The optimal retention levels are shown in Figure 3. The probabilities of absolute ruin with optimal reinsurance and the probabilities of absolute ruin without reinsurance are shown in Figure 4.

Figures 1 and 2 display the difference of the retention level and the probability of absolute ruin between exponential claims and Pareto claims with the same mean value. We see from Figure 1 


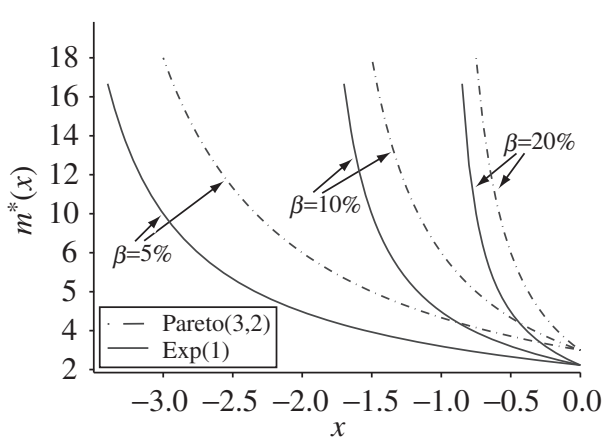

(a)

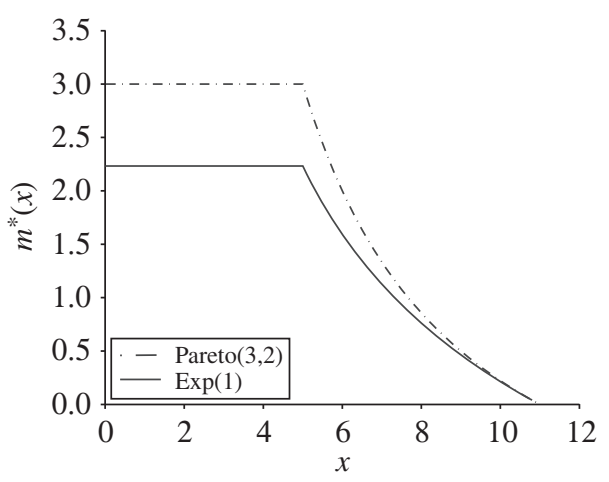

(b)

Figure 1: Optimal retention levels with (a) $\alpha=5 \%, \beta=5 \%, 10 \%, 20 \%, x<0$, and (b) $\alpha=0.05$, $\beta=\infty, x \geq 0$.

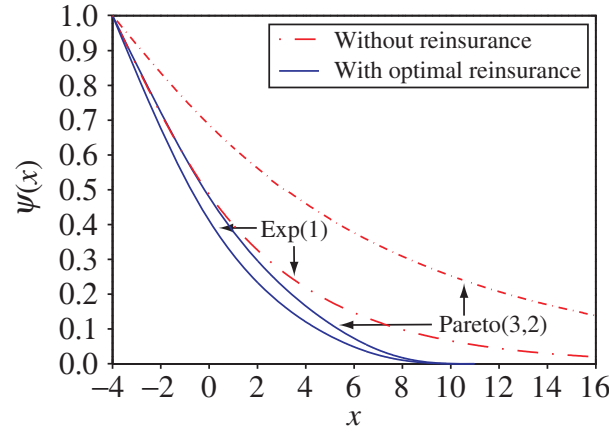

(a)

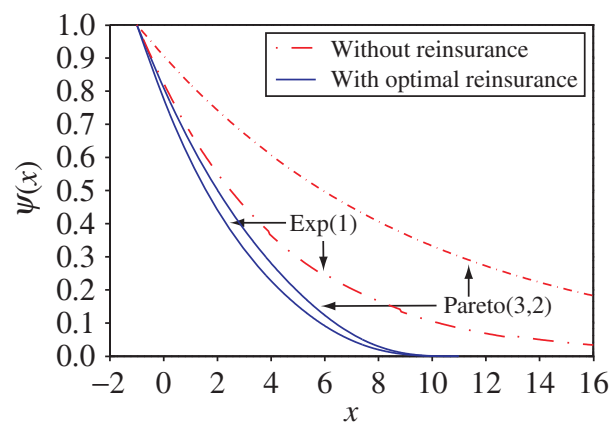

(c)

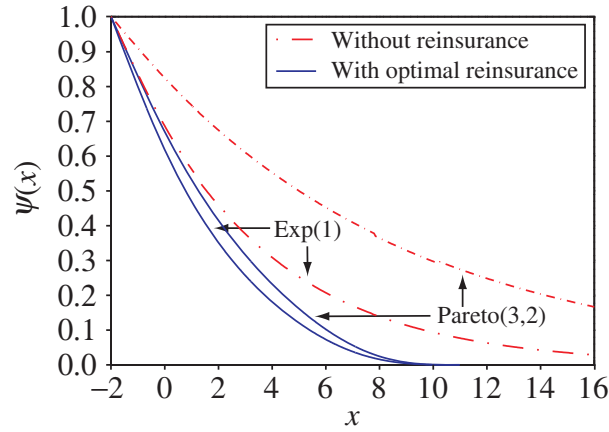

(b)

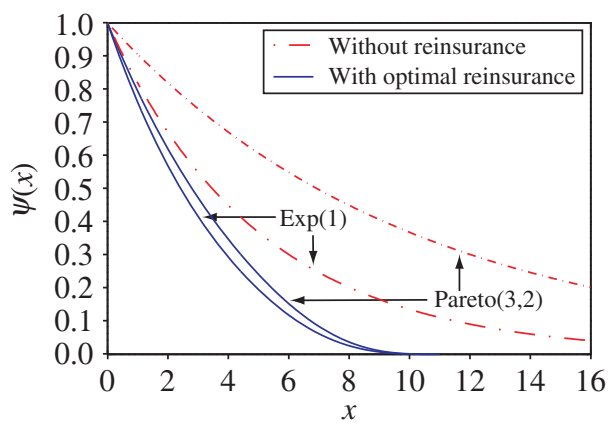

(d)

Figure 2: Probabilities of absolute ruin with $\alpha=5 \%$ and (a) $\beta=5 \%$, (b) $\beta=10 \%$, and (c) $\beta=20 \%$. (d) The probability of ruin for $\alpha=5 \%$ and $\beta=\infty$.

that the optimal retention level for Pareto claims is always higher than that for exponential claims. That is to say, to achieve the optimization, the insurer will have to take more risk for heavy-tailed claims. From Figure 2, we see that the probability of absolute ruin for Pareto claims is always greater than that for exponential claims, which is consistent with our intuitions. 


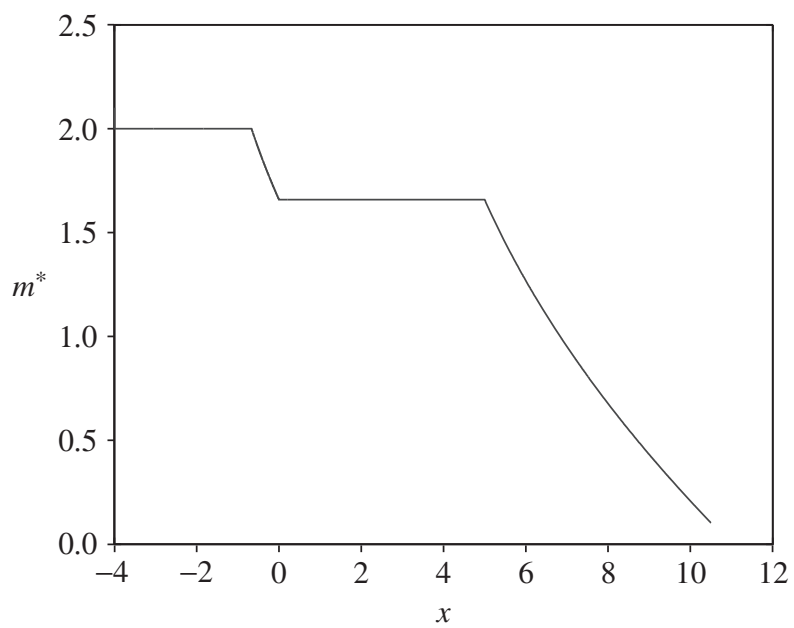

Figure 3: Optimal retention level for the uniform case with $\alpha=5 \%$ and $\beta=5 \%$.

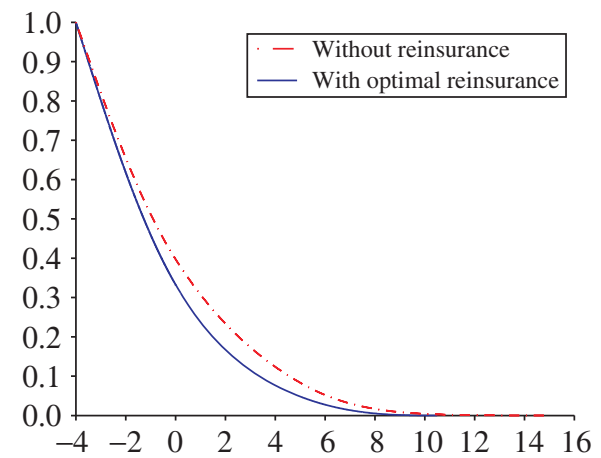

(a)

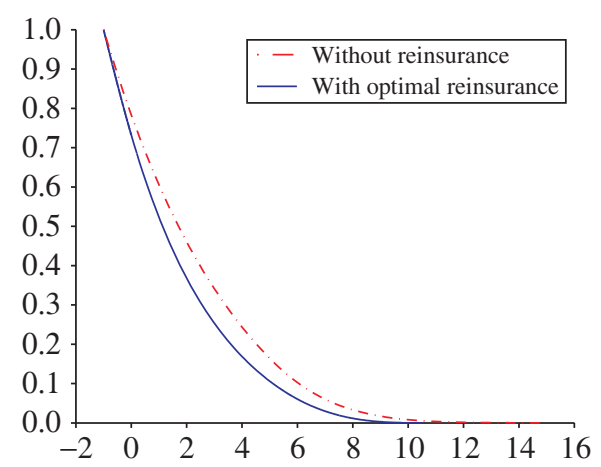

(c)

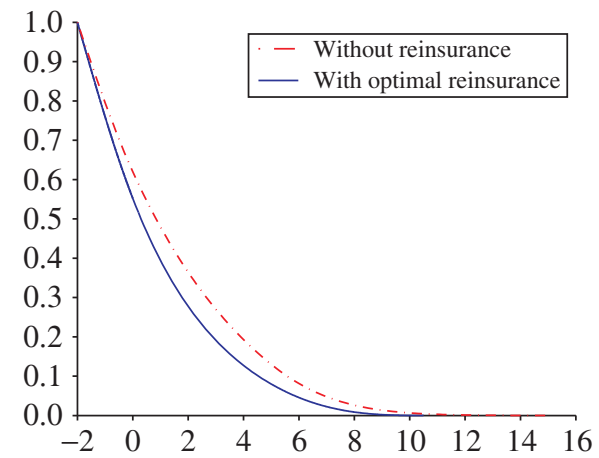

(b)

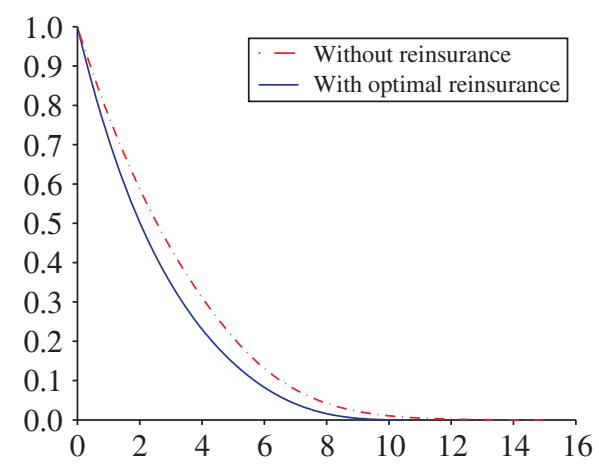

(d)

Figure 4: Probabilities of absolute ruin for the uniform case with $\alpha=5 \%$ and (a) $\beta=5 \%$, (b) $\beta=10 \%$, (c) $\beta=20 \%$, and (d) $\beta=\infty$. 
In addition, we can see that the probability of absolute ruin is significantly reduced by taking the optimal reinsurance policy. The degree of the reduction of absolute ruin probability for Pareto claims is larger than that for exponential claims.

From Figures 2 and 4, we see that the probability of absolute ruin can be reduced significantly by the optimal reinsurance policy when the initial surplus $x$ is between 4 and 10, which means that reinsurance is a very effective tool for middle-size insurance companies to reduce their risks.

From Figures 1 and 3, we see that the retention levels increase to $M$ (the maximal value of a claim size) or are equal to $M$ as the surplus decreases to the critical level $\tilde{d}$ at which absolute ruin will occur. This means that insurers should keep more risks when their surplus is at low levels.

Furthermore, from Figures 2 and 4, we see that, as the borrowing interest force $\beta$ increases, reinsurance has a significant influence in reducing the probability of absolute ruin. This has an intuitive explanation. In principle, risk can be reduced by either borrowing or reinsurance. However, as borrowing interest rates increase, the cost to reduce risk by borrowing increases. Hence, reinsurance plays a more important roles in reducing risk when borrowing interest rates are high.

\section{Acknowledgements}

The authors thank the anonymous referee for the careful reading and the useful comments and suggestions that improved the presentation of this paper. The first author is thankful for the support from the Chinese Social Science of State Education Ministry (12YJC630322) and the MOE Project of Key Research Institute of Humanities and Social Sciences at Universities (11JJD790053). The second author acknowledges the support from the Natural Sciences and Engineering Research Council (NSERC) of Canada.

\section{References}

Asmussen, S. And Albrecher, H. (2010). Ruin Probabilities, 2nd edn. World Scientific, Hackensack, NJ.

Asmussen, S., HøjgaArd, B. And TAKsar, M. (2000). Optimal risk control and dividend distribution policies. Example of excess-of-loss reinsurance for an insurance corporation. Finance Stoch. 4, 299-324.

Borovkov, A. A. (2009). Insurance with borrowing: first- and second-order approximations. Adv. Appl. Prob. 41, $1141-1160$.

CAI, J. (2007). On the time value of absolute ruin with debit interest. Adv. Appl. Prob. 39, 343-359.

Cai, J., Feng, R. AND Willmot, G. E. (2009). On the expectation of total discounted operating costs up to default and its applications. Adv. Appl. Prob. 41, 495-522.

Embrechts, P. And Schmidli, H. (1994). Ruin estimation for a general insurance risk model. Adv. Appl. Prob. 26, 404-422.

Fleming, W. H. And Soner, H. M. (1993). Controlled Markov Processes and Viscosity Solutions. Springer, New York.

Gerber, H. U. AND YANG, H. (2007). Absolute ruin probabilities in a jump diffusion risk model with investment. N. Amer. Actuarial J. 11, 159-169.

HøjgaARD, B. AND TAKsar, M. (1998). Optimal proportional reinsurance policies for diffusion models. Scand. Actuarial J. 2, 166-180.

LUO, S. AND TAKSAR, M. (2011). On absolute ruin minimization under a diffusion approximation model. Insurance Math. Econom. 48, 123-133.

Meng, H. And Zhang, X. (2010). Optimal risk control for the excess of loss reinsurance polices. ASTIN Bull. 40, 179-197.

Pachpatte, B. G. (1973). A note on Gronwall-Bellman inequality. J. Math. Anal. Appl. 44, 758-762.

SchmidLi, H. (1994). Diffusion approximations for a risk process with the possibility of borrowing and interest. Commun. Statist. Stoch. Models 10, 365-388.

SchmidLi, H. (2001). Optimal proportional reinsurance policies in a dynamic setting. Scand. Actuarial J. 1, 55-68.

TAKSAR, M. I. (2000). Optimal risk and dividend distribution control models for an insurance company. Math. Methods Operat. Res. 51, 1-42. 
TAKSAR, M. I. AND MARKusSEn, C. (2003). Optimal dynamic reinsurance policies for large insurance portfolios. Finance Stoch. 7, 97-121.

Zhang, X., Zhou, M. AND Guo, J. Y. (2007). Optimal combinational quota-share and excess-of-loss reinsurance policies in a dynamic setting. Appl. Stoch. Models Business Industry 23, 63-71.

Zhou, M. And Yuen, K. C. (2012). Optimal reinsurance and dividend for a diffusion model with capital injection: variance premium principle. Economic Modelling 29, 198-207.

ZHU, J. AND YANG, H. (2008). Estimates for the absolute ruin probability in the compound Poisson risk model with credit and debit interest. J. Appl. Prob. 45, 818-830. 